\title{
Nuevos desafíos para la formación del profesional de la información frente al surgimiento de la cibersociedad: un enfoque de competencias
}

\author{
Johann Pirela Morillo \\ Tania Peña Vera
}

El mundo actual plantea nuevos y complejos retos para formar a los profesionales vinculados con la gestión del 7 de mayo de 2004. Artículo aceptado: 17 de noviembre de 2004. conocimiento; en función de lo cual se ha desarrollado una investigación documental cuyos objetivos apuntan a profundizar teóricamente sobre la concepción educativa basada en competencias; caracterizar dicho esquema de formación en la enseñanza de las ciencias de la información, y proponer competencias para el profesional de la información a la luz del planteamiento de la cibersociedad. Se concluye que la concepción basada en competencias hace más significativa la formación y capacitación de profesionales, y que lo mismo sucede con la contextualización y aplicación de los nuevos conocimientos, la ejercitación del autoaprendizaje y la socialización del individuo. Se observa también un desplazamiento de la visión instrumental-técnica y documental-informacionalista del objeto de estudio, y por ende de la profesión, hacia una visión compleja, integradora, holística e informacional-cognitiva, lo cual 
amplía las funciones de bibliotecólogos, archivólogos y documentalistas, entendidos como profesionales del conocimiento. Además se proponen nuevos perfiles profesionales, tales como: ingeniero de la información, gestor del capital intelectual, mediador de información y comunicador de conocimientos registrados en formatos impresos y digitales.

Palabras clave: Competencias profesionales; Formación del profesional de la información; Sociedad del conocimiento y la comunicación; Aprendizajes experienciales.

\section{ABSTRACT}

New challenges for the formation of the information professional put before the emergence of cibersociety: a focus on competence

Johann Pirela-Morillo y Tania Peña-Vera

Today's world raises new and complex challenges for the formation of professionals linked to knowledge management; thus, a literature research intends to go deeper into the theoretically aspects of the educational concept of competence; to characterize such a scheme of education for the teaching of information sciences; and to propose abilities for the information professional in the context of cibersociety. It is concluded that the idea based on abilities or competence makes the education and training of professionals more significant, and that contextualization and application of new knowledge, self-learning and the socialization of the individual point in the same direction. A displacement of the emphasis on the purely technical-instrumental and documentary-informational is observed, and a tendency towards a complex, integrative holistic and cognitive-informational vision of things. Such a perception would widen the scope of librarians, archivists and documentalists as knowledge professionals. New professional profiles are also proposed: information engineer, manager of intellectual capital, information middle-man, and communicator of knowledge registered in digital and printed formats.

Keywords: Professional competence; Education of information professionals; Knowledge and communication society; Empirical learning. 
$\mathrm{E}^{1}$ artículo recoge planteamientos sobre la necesidad de desarrollar para los profesionales de la información, nuevas competencias, las cuales se están desplazando de la gestión de información hacia la comunicaciónmediación del conocimiento, asumiendo el tránsito de una sociedad de la información hacia una sociedad del conocimiento, de la comunicación y el ingenio humano, y de la cibersociedad, que les plantea nuevos desafíos a la formación de estos profesionales.

Los planteamientos realizados se sustentan en una investigación documental y un análisis crítico de enfoques y tendencias sobre los rasgos que definen a la sociedad actual y su relación con el modelo de formación basado en competencias. Los objetivos de la investigación apuntan en tres direcciones: 1 profundizar teóricamente acerca de la concepción educativa basada en competencias; 2 caracterizar el enfoque de competencias en la enseñanza de las ciencias de la información y 3 proponer competencias para el profesional de la información a la luz del planteamiento de la cibersociedad.

Se concluye que la formación de estos profesionales debe orientarse hacia la educación de usuarios para la interacción tecnoinformativa y la construcción del conocimiento como base de la expansión y multiplicación de sus facultades intelectuales; ya que en la cibersociedad es el sujeto y sus procesos de aprendizaje los que adquieren centralidad por encima de las tecnologías, las cuales son consideradas como un elemento activador y potenciador de procesos cognitivos. Sobre esta base, se proponen como resultado competencias específicas que debe asumir el profesional de la información en la cibersociedad.

\section{i. Demandas emergentes \\ DEL CONTEXTO SOCIAL: LA NECESIDAD DE UNA \\ FORMACIÓN CENTRADA EN COMPETENCIAS}

Las crecientes y complejas demandas de la sociedad global que devienen, en parte, de los avances tecnológicos y las transformaciones económicas a las que hoy asistimos, imponen a las organizaciones, y por ende a la fuerza de trabajo que las anima, la búsqueda de mayor competitividad. Por tal razón se han incrementado los niveles de exigencia frente a los sistemas de educación y capacitación de los recursos humanos con la intención de que los individuos puedan enfrentar los cambios, adaptarse rápidamente a las nuevas condiciones y en general mejorar la calidad de su desempeño en el entramado económico que sustenta nuestro sistema-mundo. 
Ibarra (2002), sostiene que los sistemas de formación y capacitación de recursos humanos están impulsando cambios tanto en las formas de organización y contenido de los programas como en los métodos de enseñanza; y su horizonte se expande hacia el hallazgo de una estrecha vinculación con las transformaciones que está experimentando la estructura productiva del mundo en relación con sus tendencias tecnológicas y los nichos ocupacionales que ofrece. Su propósito es que la fuerza laboral desarrolle una mayor destreza y pertinencia en las actividades que realiza.

También señala que los escenarios emergentes demandan especial atención en la formación de los recursos humanos, la cual ha de caracterizarse por ser integral, permanente y orientada a elevar los niveles de productividad y competitividad de los individuos. Asimismo debe enfatizarse el desarrollo de las tres capacidades básicas señaladas por la Organización para la Cooperación y el Desarrollo Económico (OCDE) (citada por Ibarra, 2002): la capacidad de innovación, esto es, la imaginación y la creatividad; la capacidad de adaptación, es decir, la adecuación rápida a los cambios; y la capacidad de aprendizaje, que es la aprehensión continua y sistemática de nuevos conocimientos.

Las condiciones de los mercados globales y los cambios tecnológicos están propiciando rupturas en los esquemas de funcionamiento tradicional de las empresas, que ya no pueden continuar siendo rígidas en sus sistemas de producción ni basarse en estructuras jerárquicas piramidales que propician la repetición y monotonía de las labores de sus trabajadores. Por el contrario, la tendencia es hacia la producción basada en las demandas y en la conformación de redes y equipos de trabajo; es decir, el achatamiento de las estructuras, la flexibilidad y el establecimiento de estrategias de mejora continua del personal de modo que éste incorpore y aporte cada vez más de sus propios conocimientos al proceso de producción, sin por ello dejar de participar en el análisis y solución de los problemas que obstaculizan el aumento de la calidad y la productividad dentro de la empresa. Lo señalado resalta la importancia que ha cobrado el aporte intelectual, creativo, e innovador de los trabajadores.

Morfín (2002) explica que esto se debe al hecho de que en la actual coyuntura la generación, transformación y difusión de la información incide notoriamente en todos los aspectos vitales de la sociedad contemporánea, y ello hace que la competitividad sólo pueda crearse y sostenerse a partir de la generación de valor agregado mediante la aplicación del conocimiento humano, que es el único elemento capaz de transformar los recursos naturales en fuentes de riqueza y bienestar.

Afirma este autor que estos cambios han iniciado una progresiva transformación de los puestos de trabajo; es decir, un desdibujamiento de las fronteras 
tradicionales de las rutinarias funciones que anteriormente realizaba la planta laboral. Hoy los procesos productivos que se desarrollan en la actualidad demandan que los trabajadores posean una combinación de competencias técnicas y académicas que los capaciten para tomar decisiones, aprender y adaptarse con facilidad a las distintas formas de organización del trabajo, además de desenvolverse ingeniosamente y mostrar habilidades múltiples y manejo de contingencias.

Todo este conjunto de exigencias se vuelca sobre el sistema educativo, el cual debe en primer lugar reconocer estas transformaciones para ajustar su orientación y propiciar la formación de individuos que desarrollen habilidades para aprender por sí mismos.

\section{SOBRE LA DEFINICIÓN DEL TÉRMINO COMPETENCIA Y SUS CARACTERÍSTICAS}

El término competencia se asocia con conceptos referidos a la calidad, el alto rendimiento, la excelencia, el desarrollo de nuevos métodos, la combinación de las exigencias entre las tecnologías y las destrezas de los trabajadores, el pensamiento creativo, la resolución de problemas con mínimos márgenes de error, el trabajo en equipo y, sobre todo, los deseos de aprendizaje siempre insatisfechos. Señala Vinent (2000) que en su semblanza etimológica la palabra competir procede del latín competere, que a su vez está compuesto por la palabra petere, que es pedir, aspirar, o tender a; y cum o com, que sugiere la idea de compañía, de compartir; por lo tanto, competere indica un aspirar o ir al encuentro de una misma cosa, un contender dos o más contrincantes para alcanzarla. En cuanto al término competencia, sostienen Muñoz, Quintero y Munévar (2001) que éste deriva de los vocablos latinos competens, competentes, que significa la aptitud legal o autoridad para resolver cierto asunto; o el que conoce o es experto o apto en cierta ciencia o materia. En líneas generales, Vásquez, Aznarán, Pérez y Alvarado (1999) consideran que la competencia plantea una situación compleja, multidimensional y cuya identificación, planificación, implementación y evaluación constituye desafíos.

Existen diversas definiciones del vocablo competencia, cuyas diferencias están en función del contexto en el que se maneje el término; sin embargo, en el ámbito laboral es donde mayormente se usa, ya que según la Organización Internacional del Trabajo (OIT) (s/f), el vocablo competencia se refiere en principio a la capacidad para el desempeño de una determinada función o actividad, de manera eficiente, eficaz y creativa; es decir, al desempeño real de un individuo basado en su instrucción y la experiencia acumulada en situaciones concretas de ejercicio ocupacional. 
En esta misma tónica, Benavides (2002) señala que las competencias son comportamientos manifiestos en el desempeño laboral que le permiten a una persona actuar eficazmente; también indica que es en esencia un comportamiento productivo observable que engloba no sólo las aptitudes sino también los conocimientos, las destrezas, las emociones y los factores de la personalidad general, amalgamados, desplegados y visibles en la práctica laboral.

Fletcher (2000) define la competencia como la aptitud de una persona para desempeñar una misma función productiva en diferentes contextos de trabajo y con base en los resultados esperados; es decir, que la evidencia de la tenencia de competencias es un desempeño consistente. En esta definición resalta de modo singular la adaptabilidad y versatilidad en la aplicación de los conocimientos, o dicho de otro modo, la capacidad del individuo para trasladar, transferir o traspolar sus conocimientos, obteniendo en cada caso altos márgenes de logro.

También la puntualiza como el conjunto de habilidades y conocimientos necesarios para enfrentar la complejidad de la agregación de valor con disminución de costo y la resolución de problemas de la organización. Masterparqua (citado por Vásquez et.al, 1999) define las competencias como las características personales (conocimientos, habilidades y actitudes) que llevan a desempeños adaptativos en ambientes significativos. (p.12)

Desde la perspectiva psicológica, existen varias definiciones del término competencia, una de ellas es la de Kane (citado por Vásquez et. al, 1999) quien la concibe como la medida en que una persona puede utilizar sus conocimientos, actitudes, aptitudes y hacer juicio, asociado a su profesión, para poder desempeñarse en forma eficaz en todas las situaciones que correspondan en el campo de su práctica profesional. (p. 13)

$\mathrm{Al}$ analizar estas definiciones puede observarse que las competencias involucran no sólo lo cognitivo e instrumental del individuo sino también lo afectivo, ello individualiza e impone un sesgo en cuanto a la manifestación de habilidades o capacidades para realizar cualquier acción, por cuanto el ingrediente emocional incide significativamente en todas las esferas del ser humano.

En cuanto a las características esenciales de las competencias, Malpica (2002) indica que en primer lugar éstas privilegian el desempeño, por cuanto éste es la expresión concreta de los saberes de un sujeto cuando realiza cualquier actividad, lo cual es más valioso que el cúmulo de conocimientos aislados que pueda poseer. Es en el desempeño donde el individuo enfrenta y resuelve situaciones reales a través de la puesta en acción de los recursos de que dispone; es decir donde traslada los conocimientos adquiridos a un plano instrumental; lo cual agudiza, por demás, su capacidad para discernir cuándo y cómo ha de usarlos adecuadamente. 
Por otra parte, las competencias conforman una totalidad, en el sentido de que en ellas convergen o se ponen en juego varios elementos relevantes ante una situación, lo cual elimina la visión atomizada en la que se pretende darle mayor valor a uno u otro tipo de conocimiento. Los elementos que intervienen o nutren una competencia tienen sentido en función del conjunto, aisladamente pierden su significado para ésta. En cada ejecución humana convergen varios constructos que no son exclusivos de ninguna de las competencias que un individuo posee, pero que pueden ser aplicados de manera distinta en diversas ocasiones.

Por último las competencias favorecen el desarrollo de mayores niveles de autonomía en los individuos, ya que les permiten hacer uso de lo que saben para mejorar su desempeño. En este sentido el sujeto fortalece su capacidad de organizar y dirigir su propio aprendizaje al enfrentar situaciones que le plantean la necesidad de integrar conceptos o conclusiones, o proponer nuevas alternativas.

Circunscribiendo el concepto al ámbito del aprendizaje, Vásquez et. al (1999) y Fletcher (2000) señalan que una competencia posee tres componentes: en primer lugar está lo conceptual, que es la especificación de lo que una persona calificada debe SABER en cuanto a los elementos teóricos básicos para ejercer adecuadamente su trabajo, más el conocimiento de las prácticas y normas establecidas que le permiten identificar y reproducir secuencias de procesos; en esencia, el saber corresponde a todo aquel conocimiento susceptible de ser medido a través de algún tipo o instrumento de evaluación. En segundo lugar la competencia incluye también lo procedimental, que es el SABER HACER; es decir, la habilidad de reproducir, en conductas laborales observables, las distinciones del conocimiento: traducir el conocimiento en acción, operacionalizar los saberes teóricos que se manejan. Y en tercer lugar viene lo actitudinal que es el SABER SER que corresponde a las habilidades personales, interpersonales o sociales y a la actitud del individuo para realizar las funciones que le competen, que incluye la honestidad, el autocontrol, el dominio de situaciones de alto riesgo, etcétera.

\section{El enfoque de competencias como modalidad educativa}

Asociando el término competencia con el entorno educativo, Malpica (2002) señala que este concepto es relativamente novedoso y se vincula con el ofrecimiento de un espacio para analizar y construir opciones educativas más acordes con las necesidades individuales y sociales de hoy, y fortalecer así la débil relación -interpretación de las necesidades de formación entre los individuos y el sector productivo. De allí que la competencia se centre en el desempeño 
y en la recuperación de condiciones concretas en que tal desempeño es relevante. Esto viene a resaltar el impacto que la educación debe causar sobre la actuación de los individuos para dejar de constituir sólo un requerimiento formal de años de escolaridad; además que permite abordar las relaciones entre los factores del contexto y las formas de organización del trabajo.

$\mathrm{Al}$ abordar la concepción educativa basada en competencias es necesario aclarar el hecho de que en el ámbito de las competencias el contexto laboral se superponga al profesional, lo cual según Tejada (1999) estriba en que la competencia comporta en sí misma un conjunto de conocimientos, procedimientos y actitudes combinados, coordinados e integrados para el ejercicio profesional; es decir, que el dominio de estos saberes hacen al individuo"capaz de" actuar con eficacia en situaciones profesionales en las que se requiere "saber hacer" y "saber estar". Se entiende entonces que las competencias profesionales propiamente dichas son aquellas que el sujeto adquiere y desarrolla durante su praxis profesional; tal y como lo señala la OIT (s/f) al definir las competencias profesionales como aquellas que son adquiridas en el ejercicio de la experiencia profesional, de manera que los profesionales competentes son los reconocidamente "buenos" en su práctica profesional, lo cual lleva implícita una evaluación positiva por parte del entorno social.

Existe pues una relación indisociable entre las competencias laborales y profesionales, las primeras facilitan la asimilación, apropiación y sistematización de la cultura organizacional en la cual se desenvuelve el individuo. Y las segundas, de acuerdo con Ortiz (s/f), se van formando en el proceso pedagógico; es decir, en el proceso de aprender la profesión, en el cual el profesional en formación transita de un estado inicial a otro final cualitativamente superior tras cumplir distintas etapas de formación y desarrollo de sus conocimientos, habilidades y valores.

De esto se deduce que si bien ambos tipos de competencias inician en contextos distintos, las unas en el ambiente de las empresas y las otras en las instituciones educativas, existe un encuentro inevitable entre ellas, además de una afectación mutua por cuanto que sólo es posible validar o garantizar un adecuado desarrollo de capacidades y destrezas en un individuo cuando se propicia una educación integral; es decir, una interacción permanente entre la teoría y la práctica, o la contrastación de elementos teóricos con las realidades productivas de las organizaciones. Es esta integración lo que favorece la formación de un trabajador altamente calificado, competente y competitivo, con un alto desarrollo de sus competencias profesionales y de su capacidad de satisfacer demandas económico - productivas.

En palabras de Huerta, Pérez y Castellanos (s/f), el propósito de la educación basada en competencias es combinar la educación y el trabajo reconociendo los aportes de la educación formal y los conocimientos, habilidades y destrezas 
adquiridos fuera de las aulas; se trata de que se establezca un equilibrio entre los elementos teóricos que se manejan y la solución de problemas específicos. El eje principal de este tipo de educación es entonces el desempeño, considerado como la materialización de los recursos cognitivos del sujeto cuando lleva a cabo una actividad, en lo cual se evidencia el uso o manejo que éste realiza sobre lo que sabe; todo ello indica que se pone un énfasis especial en el empleo que se haga de los conocimientos, más que en la sola tenencia de ellos.

La incorporación de competencias en el ámbito educativo, según indica Torrado (2000), es algo en lo que se ha venido trabajando desde hace algún tiempo, pero además existen experiencias en algunos países como Reino Unido, Canadá, Australia, y Colombia, entre otras, que demuestran los beneficios que se obtienen al aplicar esta forma o esquema de aprendizaje. Lo central de ello estriba en propiciar que la educación sirva como un instrumento efectivo para desarrollar los conocimientos, las destrezas, las habilidades y las actitudes básicas que les permitan a los individuos desempeñarse autónoma, productiva y constructivamente en los diferentes espacios de la sociedad.

Por otra parte basar la formación de individuos en torno al desarrollo de competencias incluye además elementos de tipo ético, pues se ha de hacer énfasis en el ¿para qué? de cada contenido impartido, y ello sin duda conduce a un proceso de reflexión y concientización por parte del sujeto, quien ha de internalizar el uso y la importancia de lo que aprende en cada situación en la que se desenvuelva profesionalmente. Este aspecto cobra mayor relevancia en el contexto universitario, ya que es en la educación superior donde ha de reforzarse aún más el sentido crítico y ético de los educandos con la intención de propiciar la formación de profesionales que se conduzcan como verdaderos agentes de cambio en la sociedad.

Esto lo corrobora Torrado (2000) quien señala que el modelo curricular por competencias demanda reorientar las prácticas educativas, además de revisar la selección y organización de los contenidos y actividades de enseñanza; es decir, se hace necesario desmontar el esquema de transmisión unilateral de conocimientos, así como la superación del aprendizaje memorístico, para abrir paso a espacios de interpretación, reflexión, experimentación y debate sobre los temas que se discutan, para resignificarlos y reapropiarlos a nivel individual y colectivo; por consiguiente, el carácter acabado de los contenidos debe ser modificado para entenderlos como constructos en permanente revisión y adecuación.

Sigue apuntando Torrado que cuando se asume el desarrollo de las competencias como el núcleo de la educación, también ha de cambiar la forma de mirar al sujeto que se educa, el cual pasa a ser un agente activo en su propia 
formación, y quien es además afectado por todo el entramado social en el que se encuentra inserto. Es necesario, pues, reforzar la construcción significativa de los conocimientos de modo que en su mente se conformen redes conceptuales que le permitan reorganizar constantemente lo que conoce, y hacer que su conocimiento sea cada vez más accesible y pertinente al ser utilizado. También es preciso propiciar en él la creación de nuevo conocimiento mediante su intercambio y socialización con otros actores con quienes habrá de implicarse ética y emocionalmente para construir colectivamente saberes, significados, realidades que transformen el entorno.

En función de lo señalado, se concluye que el modelo educativo basado en competencias se orienta a la formación de profesionales que conciban el aprendizaje como un proceso abierto, flexible y permanente, y que trasciende el espacio en el que se recibe la educación formal. En esencia esta modalidad promueve la combinación e integración de momentos del aprendizaje académico implicados con situaciones de la realidad profesional, con la finalidad última de elevar el nivel de la formación que reciben los individuos.

\section{Clasificación de las competencias}

Existen varias clasificaciones de las competencias si tomamos en cuenta las perspectivas teóricas que manejan los autores. Según Fletcher (2000) y Benavides (2002) las competencias se clasifican en tres grupos:

- Competencias básicas: son las que otorgan conocimientos básicos generales para entender el mundo y participar en la sociedad; por ejemplo la lecto-escritura, operaciones matemáticas elementales, habilidades para establecer relaciones interpersonales y de socialización. Tales competencias se dividen en tres grandes segmentos: habilidades básicas, habilidades del pensamiento y cualidades personales.

- Competencias genéricas: son las habilidades o características requeridas por los individuos que pueden generalizarse en una empresa, entidad, consorcio o estado. Su finalidad es permitirle a los trabajadores ser útiles en sus equipos de trabajo y desenvolverse con alto desempeño, mediante el fortalecimiento de la identidad como miembros de estos entes; es decir, se basan en la orientación organizacional.

- Competencias específicas: llamadas también gerenciales u ocupacionales. Son los comportamientos competitivos distintivos frente a productos, servicios, ventas o posicionamientos que hacen referencia al uso y mejoramiento del desempeño personal en el manejo de recursos financieros, tecnológicos, de información, etcétera, que responden a la especialidad 
laboral de cada individuo, por cuanto contemplan el conjunto de atributos personales visibles que se aportan al trabajo; es decir, los comportamientos necesarios para lograr un desempeño idóneo y eficiente.

En esta clasificación se puede evidenciar un incremento progresivo del saber hacia áreas concretas, pues se observa un acercamiento que parte de lo general y avanza hacia lo particular. Esto se corresponde de algún modo con la evolución orgánica que experimenta el aprendizaje del individuo, el cual va asumiendo mayores niveles de complejidad en la medida que se incrementa su madurez intelectual.

Al vincular las competencias con el ámbito educativo se puede manejar el término de la misma manera como se hace en la industria, en la cual se asocia con la búsqueda de un perfil competitivo y valioso para el cliente; en la esfera educativa también se trata de hacer más y lo mejor posible que los otros para obtener liderazgo en el mundo del trabajo, lo cual se encuentra, según Fletcher (2000) en el seno mismo de la reflexión educativa contemporánea.

Si en el plano laboral la orientación tradicional de la formación de los individuos está sufriendo importantes ajustes que se orientan hacia la valoración de lo humano y social por encima de lo laboral y económico, también, y más aún en el contexto educativo, se ha venido incrementando el interés hacia los valores y satisfacciones del individuo, quien pasa a ocupar el centro de atención del proceso, visto ya no como ejecutor de órdenes o tareas que conllevan a la producción de bienes y servicios, sino como ente holístico en el que convergen conocimientos y sentimientos que no pueden funcionar desarticuladamente.

Señalan Vásquez et. al (1999) que la división internacional del trabajo que se ha producido por influencia de la globalización ha impuesto una condición tecnológica que se centra en el manejo, el procesamiento, el acceso y la distribución de la información; lo cual ha propiciado que las demandas de los puestos de trabajo experimenten significativas transformaciones que se evidencian en un mayor énfasis en la transferencia de los conocimientos, la preferencia por el trabajo en equipo y la sustitución del esfuerzo físico por el cerebral. Esto a su vez ha impuesto nuevas demandas en la formación de los recursos humanos, que ha de centrarse más en la ocupación y el desarrollo de competencias laborales.

Para estos autores la formación basada en competencias parte de vincular el mundo académico con el laboral, esto con el objeto de mejorar la calidad y eficiencia en el desempeño a partir de una formación de base amplia que reduzca el riesgo de obsolescencia de los conocimientos. Por tal razón el énfasis de los currícula basados en competencias es la formación de un profesional que cuente con competencias profesionales específicas que le 
permitan asumir sus responsabilidades, justificar sus decisiones y ejecutarlas con propiedad haciendo uso del cúmulo de conocimientos teóricos- instrumentales. De igual modo será necesario desarrollar competencias para hacer un aprendizaje permanente y adaptarse al cambio con base en el autoaprendizaje contextualizado y la ejercitación del pensamiento crítico.

\section{LA FORMACIÓN ACADÉMICA-PROFESIONAL EN CIENCIAS DE LA INFORMACIÓN} A PARTIR DEL ENFOQUE DE COMPETENCIAS

Aplicar el enfoque de competencias resulta en este momento de fundamental importancia sobre todo en áreas de conocimiento como las ciencias de la información, cuyo objeto de estudio debe concebirse como una coordenada que atraviesa los diversos ámbitos de las ciencias, las artes y la tecnología. Plantear el enfoque de competencias como modelo curricular de la formación del profesional de la información implica asumir la presencia de una sociedad reticular, en la cual adquiere un carácter estratégico el proceso de comunicación-mediación que incluye la organización, la representación, la difusión y la producción innovativa del conocimiento soportadas en tecnologías digitales e interactivas, que ofrecen nuevas posibilidades de expansión a la inteligencia humana.

En palabras de Wolton (2000), los dos tipos de mediadores fundamentales de la información y el conocimiento de este nuevo siglo serán por un lado los bibliotecólogos, archivólogos y documentalistas, y por el otro los periodistas, porque estos profesionales trabajan con los dos tipos de información más importantes que fluyen en estos momentos por los diferentes puntos de la Red que requieren organización, sistematicidad y articulación: la denominada información-conocimiento y la información-noticia.

Por ello se requiere que los bibliotecólogos y archivólogos como profesionales de la información asuman un nuevo papel mediador que incluya no sólo los procesos de organización y representación del conocimiento, sino también la educación de usuarios para la interacción tecnológica, lo que a su vez supone desarrollar habilidades para la búsqueda, filtrado, procesamiento y construcción del conocimiento, mediante las tecnologías telemáticas e interactivas. Este desafío le impone a estos profesionales, una subordinación al paradigma tecnocultural que propone Sodré (1998) y (2000), en el cual son otras las categorías que explican los procesos de comunicación e información y el sujeto pasa a ser, además de un ser psico-social, un bios virtual con estructuras cognitivas complejas favorecedoras de nuevos mapas de pensamiento y acción como producto de las tecnointeracciones producidas entre ellos y las tecnologías. 
Se requiere entonces una formación basada en competencias para el desarrollo de acciones comunicativas-mediadoras soportadas en tecnologías telemáticas, pero la pregunta ahora es: ¿Cuáles son las "nuevas” competencias que deben desarrollar y aplicar los profesionales de la información frente al desafío y características de la cibersociedad?

Para responder la pregunta es necesario ubicar la emergencia de una realidad social-cultural que no está centrada en la información sino más bien en el conocimiento y la comunicación, lo cual plantea un desplazamiento de las funciones y roles del profesional, quien más que un gestor de información pasa a ser un comunicador-mediador creativo de conocimientos contextualizados en torno a las necesidades de aprendizaje de los individuos para que éstos puedan expandir su arquitectura cognitiva, resuelvan problemas y tomen decisiones. (Abram, 1999).

En el fondo este desplazamiento obedece al tránsito de la sociedad de la información a la del conocimiento y la comunicación: la cibersociedad, sustentada en la metáfora de las redes (Castells, 1999), y no sólo redes electrónicas, sino humanas, sociales y organizacionales.

Aunque las expresiones sociedad de la información y sociedad del conocimiento son abordadas en la literatura especializada en ciencias de la información y la comunicación como términos sinónimos, y en otros casos aparecen como nociones integradas, ya que se prefiere hablar de sociedades de la información y el conocimiento, consideramos que entre estas dos expresiones hay diferencias importantes dadas por los rasgos distintivos que caracterizan los conceptos información, conocimiento y comunicación.

\subsection{Rasgos explicativos del paso de la sociedad de la información} a la sociedad del conocimiento y la comunicación

El tránsito de la sociedad de la información a la sociedad del conocimiento y la comunicación es posible caracterizarlo a partir de dos metáforas señaladas por Galindo (1998) y (2000): la verticalidad y la horizontalidad. En la sociedad de la información, simbolizada mediante la verticalidad, las características esenciales son, según Pineda et al (2003), la circulación y flujos de datos disponibles para el individuo a través de las TIC; pero apenas son los elementos activadores del proceso de conocimiento y de aprendizaje en su totalidad, el cual busca avanzar hacia la consolidación de nexos y relaciones entre las personas y sus epistemes a través de las computadoras, los hipermedios y las redes (horizontalidad), con lo cual entraríamos a la sociedad de la comunicación para desembocar en el futuro en un contexto social que se valga de las tecnologías de información y comunicación para fortalecer el aprendizaje y el ingenio humano Tapscott (1999), e inaugurar así la posibilidad de una era de la "inteligencia interconectada". 
La diferencia importante que existe entre la información y el conocimiento, en el contexto de lo que Silvio (1993) llama "compleja trayectoria cognitiva”, fue planteada ya por Páez (1992), cuando recurrió al símbolo de la pirámide para señalar que los datos son la materia prima de la información y por tanto constituyen la base de la pirámide; la información es la integración de los datos con un significado definido, y el conocimiento implica un carácter comprensivo de datos e información y favorece una intervención mucho más efectiva de los contextos.

Algunos de los rasgos generales que surgen en la sociedad del conocimiento y la comunicación y que difieren de la sociedad de la información, pueden enumerarse como sigue:

1. La sociedad de la información está organizada por relaciones y estructuras rígidas entre sus actores, y sólo una parte de ellos pueden desarrollar la creatividad porque la información fluye en un solo sentido. La sociedad del conocimiento y la comunicación, la cibersociedad, tiene una estructura mixta, constituida por formas horizontales que contrarrestan las verticales y permiten acuerdos entre pares, de allí que esta sociedad se organice sobre la base de comunidades de conocimiento. Galindo (2000) y Pineda (2003).

2. La sociedad de la información remite al comando y control de la información, mediante sistemas que se resumen en la aplicación de programas computacionales lógico-matemáticos. La sociedad del conocimiento y la comunicación fomenta la relación con el alter, el "otro"; por lo que remite no sólo a sistemas lógicos y matemáticos, sino también a sentimientos, emociones y mundos de vida reales y virtuales. (Borgucci, 1999). Esto indica que es el ser humano quien adquiere centralidad por encima de la tecnología, ésta última un elemento potenciador de las relaciones sociales, el aprendizaje y la construcción del conocimiento.

3. La sociedad del conocimiento y la comunicación es un espacio social plural, por ello es una "sociedad de los saberes para todos y por todos" (Mattelart, 2003), lo cual implica no sólo la dimensión humana del conocimiento, sino la pluralidad de los saberes y sus protagonistas: los saberes fundamentales o sabios, los saberes aplicados de expertos, los saberes ordinarios surgidos de las múltiples vivencias de la cotidianidad. Estas concepciones proponen la organización social basada en el uso de las tecnologías para lograr procesos más democráticos.

4. En la sociedad del conocimiento y la comunicación surgen nuevas formas de producción, procesamiento y aprendizaje de la información y construcción del conocimiento. Caballero (2000) plantea que emergen nuevas lógicas discursivas de consenso y disenso; lo que requiere disponer de renovados 
filtros cognitivos para ver, aprehender, comprender y materializar el mundo objetivo, expresado en pantallas y realidades digitales; ello implica que el sujeto está lejos de ser un mero operario y puede convertirse en "gerente del conocimiento”, capaz de dirigir y controlar los sistemas electrónicos para extender y multiplicar sus facultades intelectuales. (Silvio, 1993).

5. En la sociedad emergente el conocimiento es un factor clave en la estrategia de desarrollo de los países, por lo que deben hacerse importantes inversiones en capital humano, en capacidades para la investigación científica y en medios teleinformáticos para facilitar la circulación y el uso social de la información y el conocimiento.

Ante estos rasgos generales distintivos del surgimiento de una nueva sociedad, se impone la necesidad de formar a los profesionales de la información sobre la base de una racionalidad comunicativa-cognitiva compleja, que trascienda la visión procedimental-instrumental y sólo tecnológica de las profesiones vinculadas con la difusión del conocimiento, para pasar a plantear una visión mucho más holística e integral de estas profesiones, articulándolas con el aprendizaje individual y organizacional y la expansión de la inteligencia humana y social.

5.2. Las nuevas competencias del profesional: del gestor de información al comunicador-mediador de conocimientos.

La sociedad del conocimiento y la comunicación plantea nuevos desafíos a la educación de los profesionales de la información. Esta sociedad, levantada sobre las metáforas de la red y la horizontalidad, debe formar profesionales a partir del enfoque de competencias que imprime mayor dinamismo y pertinencia a los modelos curriculares, ya que proponen una educación entendida no como transmisión de contenidos semánticos, sino como la construcción y el desarrollo de procesos de pensamiento, habilidades y destrezas generales y específicas de las profesiones.

En este sentido, algunas de esas "nuevas" competencias (conocimientos, habilidades, destrezas y actitudes) que deben desarrollar los profesionales de la información giran en torno a la comunicación-mediación de la información y su impacto en el enriquecimiento cognitivo del individuo y la sociedad, para lo cual hay que asimilar conocimientos teórico-prácticos, integrarlos y/o vincularlos con otros campos del saber, además de aplicar métodos de investigación para generar su propio conocimiento. (Benitez y Miranda, 2002).

De este modo están surgiendo nuevos perfiles de competencias para los profesionales de la información, impregnados de nuevas concepciones y roles que se deben desempeñar, entre los cuales están los de ingeniero de la información, 
gestor del capital intelectual, mediador de información y comunicador de conocimientos registrados en formatos impresos y digitales. (Benitez y Miranda, 2002 y Peña y Pirela, 2002).

Igualmente, Muñoz (1998), Muñoz y Rubiano (1998), Cornella (1999), Gómez-Fernández (2002) y Cano (2002), sostienen que entre las nuevas competencias que debe desarrollar el profesional de la información en la actualidad están: la búsqueda electrónica de información; la creación y expansión de posibilidades para acceder a la información y al conocimiento que están disponibles en documentos impresos y digitales; el aprendizaje permanente y la innovación; la actitud investigativa y el énfasis en el diseño y la producción de servicios cada vez más personalizados y soportados por las tecnologías teleinformáticas.

Sánchez-Vegas (2003), también se ha referido a la necesidad de desarrollar nuevas competencias para un profesional emergente y llega a plantear la construcción de arquitecturas de conocimiento soportadas en sistemas tecnológicos expertos como la función medular de estos profesionales en la actualidad. La idea que subyace en el planteamiento es que se asiste al paso de la arquitectura de la información a la arquitectura del conocimiento, en donde, según la autora, van implícitos procesos de transfiguración de la información, por lo que recurre al concepto de infoarquitectura como el proceso de organización del conocimiento -sus procesos y estructuras- en un continuum que va de lo tácito a lo explícito y viceversa; y de integración de los distintos productos y servicios, con mediación tecnológica, en consonancia con los principios de la Organización que Aprende.

Junto a estas competencias de carácter teórico-conceptual y práctico, se proponen otras más de carácter actitudinal y de nuevas características personales para ejercer la profesión, entre las que se mencionan el compromiso personal-social para con la organización donde se trabaja y para con los usuarios; el liderazgo, la cooperación y la facilidad de comunicación e interacción con otros, lo cual facilita el trabajo en equipos multi y transdisciplinarios; y la actitud de investigación y actualización permanente, que concibe el acto investigativo como una estrategia fundamental para enriquecer su acción en diversos ámbitos de trabajo y le permite su propio conocimiento.

En esencia lo que se pretende es modelar un nuevo profesional de la información que responda adecuadamente a la realidad de este siglo XXI, para lo cual es necesario que tal profesional incorpore elementos de innovación que contribuyan a replantear modelos y estilos de gestión tradicionales, que si bien respondieron a momentos históricos particulares hoy se muestran agotados y obsoletos ante el avasallante progreso científico y tecnológico que plantea nuevas y complejas necesidades informacionales y cognitivas. (Peña y Pirela, 2002). 
Para tener una visión sucinta de las nuevas competencias del profesional de la información, presentamos una matriz que recoge las competencias en relación con cada uno de los elementos que las conforman:

Matriz relacional de competencias propuestas para el profesional de la información

\begin{tabular}{|c|c|c|}
\hline $\begin{array}{c}\text { Competencias } \\
\text { teórico-conceptuales } \\
\text { (Saber- Saber conocer) }\end{array}$ & $\begin{array}{l}\text { Competencias } \\
\text { procedimentales } \\
\text { (Saber hacer) }\end{array}$ & $\begin{array}{l}\text { Competencias } \\
\text { actitudinales } \\
\text { (Saber ser) }\end{array}$ \\
\hline $\begin{array}{l}\text { 1. Conocer los fundamentos } \\
\text { teóricos y epistemológicos } \\
\text { de las ciencias de la } \\
\text { información. }\end{array}$ & $\begin{array}{l}\text { 1.1. Aplicar hábilmente los } \\
\text { constructos teóricos y } \\
\text { epistemológicos de las } \\
\text { ciencias de la información } \\
\text { en situaciones reales de } \\
\text { trabajo como profesional de } \\
\text { la información. }\end{array}$ & $\begin{array}{l}\text { 1.1.1. Cultivar el espíritu } \\
\text { crítico, la curiosidad, la } \\
\text { acusiocidad y la exhaustivi- } \\
\text { dad, aspectos esenciales de } \\
\text { la actitud hacia el } \\
\text { conocimiento y la } \\
\text { investigación. }\end{array}$ \\
\hline $\begin{array}{l}\text { 2. Manejar teorías y modelos } \\
\text { gerenciales que permitan el } \\
\text { abordaje conceptual de } \\
\text { problemas existentes en el } \\
\text { contexto de las unidades de } \\
\text { información. }\end{array}$ & $\begin{array}{l}\text { 2.1. Generar tecnologías } \\
\text { propias para la solución de } \\
\text { problemas puntuales. } \\
\text { 2.2. Formular políticas, } \\
\text { programas y proyectos, que } \\
\text { incluyan la implementación } \\
\text { de centros y servicios de } \\
\text { información físicos y } \\
\text { digitales. }\end{array}$ & $\begin{array}{l}\text { 2.1.1 Desarrollar las actitudes } \\
\text { de liderazgo sobre la base } \\
\text { del respeto, la tolerancia y la } \\
\text { cohesión entre personas y } \\
\text { grupos. }\end{array}$ \\
\hline $\begin{array}{l}\text { 3.a. Conocer las teorías y los } \\
\text { métodos para la gestión del } \\
\text { conocimiento. } \\
\text { 3.b. Describir la historia de los } \\
\text { sistemas, procesos y } \\
\text { productos de comunicación- } \\
\text { mediación del conocimiento. }\end{array}$ & $\begin{array}{l}\text { 3.1. Agregarle valor a la } \\
\text { información para convertirla } \\
\text { en metainformación y } \\
\text { conocimiento. } \\
\text { 3.2. Construir y diseñar } \\
\text { metalenguajes. (tesauros, } \\
\text { taxonomías, sistemas y } \\
\text { redes semánticas) }\end{array}$ & $\begin{array}{l}3 \text { Interiorizar una visión del } \\
\text { conocimiento como elemen- } \\
\text { to medular de la sociedad y } \\
\text { la cultura, y como factor pri- } \\
\text { mordial del desarrollo perso- } \\
\text { nal-profesional y social. }\end{array}$ \\
\hline
\end{tabular}


4. Conocer los sistemas telemáticos usados para la gestión del conocimiento.

5. Comprender las teorías y metodologías existentes para el estudio de las necesidades y los perfiles de los usuarios.

6. Identificar los procesos de p decir, aque llos que conducen al metaconocimiento (aprender a aprender)

7. Conocer la legislación que regula la producción y el acceso al conocimiento existente en el ciberespacio.
4.1. Representar conocimientos mediante recursos telemáticos.

4.2. Construir y diseñar arquitecturas de conocimiento, soportadas en sistemas tele-informáticos. 4.3.Monitorear permanentemente los cambios y las innovaciones tecnológicas que se dan en el entorno.

4.4.Aplicar métodos de medición del conocimiento. (bibliometría, informetría y cienciometría

5 Diseñar creativamente pro ductos y servicios informacionales no convencionales, adaptados a los perfiles de necesidades y características de usuarios específicos.

5.2. Educar a los usuarios para el fomento del aprendizaje tecnológico-informativo y la inteligencia investigativa.

5.3. Interactuar con individuos y grupos de trabajo.

6.1.Transferencia de procesos de pensamiento en la representación, organización, y sistematización de información.

7.1. Aplicar la legislación que rige la producción y el acceso al conocimiento en el ciberespacio.
$4 \quad$ Desarrollar actitudes pa ra el desarrollo del pensamiento lógico, de deducción e inducción, como base de la organización, análisis y síntesis del conocimiento, mediante la utilización de medios tecnológicos.

4.2.1. Fomentar la actitud positiva y creadora acerca del uso de las tecnologías como medios para gestionar el conocimiento.

4.3.1. Asumir una actitud de vigilia y alerta acerca de los cambios que se dan en el entorno.

4.4.1. Valorar la producción de conocimiento como elemento de producción científica que es necesario medir.

5.1.1. Consolidar una actitud positiva hacia el ser humano como eje de las funciones y procesos que desempeñan las unidades y servicios de información.

5.2.1. Enseñar a otros las estrategias y rutas de búsqueda y construcción del conocimiento mediante las tecnologías telemáticas.

5.3.1. Cultivar una actitud posi$t$ y proactiva hacia el traba jo de servicio social, y las relaciones sociales y grupales

6.1.1. Apertura hacia el aprendizaje, reaprendizaje y autoaprendizaje permanente; fomento del pensamiento lateral y estratégico

7.1.1. Asumir una dimensión ética en el manejo y acceso a la información soportada en medios digitales.

Fuente: elaboración propia 
La investigación emprendida sobre los nuevos desafíos que el actual mundo globalizado impone a los profesionales de la información conduce a generar las siguientes conclusiones:

1. Las complejas demandas sociales, científicas y tecnológicas demandan una educación centrada en el desarrollo de competencias, sobre todo la educación vinculada con áreas de conocimiento, tan decisivas en estos momentos, como la información y la comunicación. Tales competencias no sólo deben abarcar los componentes teórico-conceptuales que explican el objeto de estudio y fundamentan la naturaleza y el alcance de las funciones profesionales; sino también los componentes procedimentales y actitudinales que guían el hacer y convierten la teoría en modos de actuar.

2. Los modelos de formación profesional basados en un enfoque de competencias requieren el replanteamiento de las prácticas educativas, además de la revisión, selección y organización de contenidos para determinar su actualidad y pertinencia, ya que se busca formar un profesional con un elevado perfil y preparado para enfrentarse con la complejidad y turbulencia presentes en los nuevos tiempos.

3. La formación académica-profesional en ciencias de la información debe transitar hacia la introducción del enfoque de competencias, lo cual se enmarca a su vez en el desplazamiento que se está dando en estos momentos de la sociedad de la información a la sociedad del conocimiento y la comunicación, entendida esta última como una sociedad que utiliza el aprendizaje permanente como una estrategia de desarrollo personal y social. Esto plantea la vuelta al sujeto, el desarrollo de su cognición y la expansión de su inteligencia, soportado todo ello en el acceso y uso social de la información mediatizada por las tecnologías telemáticas.

4. Ante esta nueva sociedad se requiere que el profesional de la información asuma un rol mediador, que incluye la contribución al aprendizaje de las personas y grupos; la creación de conocimiento útil basado en información, y la creación de contenidos digitales sobre la base de criterios de utilidad, accesibilidad y calidad, pero sin dejar de lado el componente ético que debe orientar la producción y el uso del conocimiento en la cibersociedad. 


\section{BiBLIOgRAFÍA}

Abram, S. (1999) "Posicionamiento de los profesionales que trabajan en las bibliotecas especializadas en la post-era de la información”, en Revista de Tecnología de la Información. No. 4 Año 1. Biblioservice C.A. Maracaibo-Venezuela. p.p. 34-38.

Benavides, O. (2002). Competencias y competitividad. Diseño para organizaciones latinoamericanas. Bogotá: McGrawHill.

Benitez, B. y Miranda, M. (2002) Competencias del profesional de la información: Un andén en el MERCOSUR. Tercer Encuentro de Directores y Segundo de Docentes de las Escuelas de Bibliotecología del MERCOSUR. En:

http:www.utem.cl/deptogestinfo/19.doc. (Consulta: 09 de noviembre de 2002)

Borgucci, E. (1999) "La gerencia del conocimiento y la ética en la organización virtual", en Revista Educación y Ciencias Humanas. No. 13. Año VIII. Maracaibo-Venezuela, pp. 61-77.

Caballero, S (2000) Organizaciones emergentes que surgen en el ciberespacio. Tesis doctoral. Caracas: CENDES.

Cano, V (2002) De bibliotecario a gestor de información. ¿Cambio de nombre o nuevas competencias? En:

http://jimmy.qmced.ac.uk/usr/imres/fulltxt/txt_vC3.htm. (Consulta 08 de noviembre de 2002).

Castells, M. (1999). La era de la información. Economía, Sociedad y Cultura. Vol. 1. Madrid: Siglo Veintiuno Editores.

Cornella, A. (1999) Extra!Net. Mensaje 440 (25-06-1999) En:

http://www.extra-net.net/articulos/en990625.htm. (Consulta: 08 de noviembre de 2002).

Gómez-Fernández, J. (2002) El profesional de la documentación. Guiones de la asignatura Midus. En:

http://www.arrakis.es/ amjg/per9.htm. (Consulta: 09 de noviembre de 2002)

Galindo, L. J. (2000). "Hacia la construcción de mundos posibles en nuevas metáforas conceptuales”, en Revista Comunicación No. 109. Ob. cit. p.p. 44-49.

Galindo, L. J. (1998) Técnicas de investigación en sociedad, cultura y comunicación. México: Pearson.

Fletcher, S. (2000) Análisis de competencias laborales. México: Panorama.

Huerta, J., Pérez, I. y Castellanos, A. (s/f). Desarrollo curricular por competencias profesionales integrales. En:

http://bibliotecnica.upc.es/Rebiun/nova/publicaciones/compe_ prof.pdf. (Consulta: 10 de agosto del 2004).

Ibarra, A. (2002). "El sistema normalizado de competencia laboral", en A. Argüelles (comp.). Competencia laboral y educación basada en normas de competencia. México D.F.: LIMUSA: 25-66.

Malpica, M. (2002). "El punto de vista pedagógico", en A. Argüelles (comp.). Competencia laboral y educación basada en normas de competencia. México D.F.: LIMUSA: 123-140. 
Mattelart, A. (2003). "La sociedad de la información. El enfrentamiento entre proyectos de investigación”, en Revista Diálogos. No 67. (Septiembre) FELAFACS. Lima-Perú. pp. 19-28.

Morfín, A. (2002). "La nueva modalidad educativa: educación basada en normas de competencia", en A. Argüelles (comp.). Competencia laboral y educación basada en normas de competencia. México D.F.: LIMUSA: 81-98.

Muñoz, C. V. (1998) El papel del gestor de la información en las organizaciones a las puertas del Siglo XXI. En: http://www.florida-uni.es/'fesabid98/Comunicaciones/m_munyoz.htm. (Consulta: 08 de noviembre de 2002).

Muñoz, J., Quintero, J. y Munévar, R. (2001). Cómo desarrollar competencias investigativas en educación. Bogotá: NOMOS.

Muñoz, M. y Rubiano, P. (1998). El bibliotecario digital: el perfil de un nuevo profesional de la información. En:

http://www.florida-uni.es/'fesabid98/Comunicaciones/m mundos.htm. (Consulta: 08 de noviembre de 2002)

Organización Internacional del Trabajo (OIT). (s/f). Glosario de términos. En:

http://www.cinterfor.org.uy/public/spanish/region/ampro/cinterfor/temas/complab/doc/otros/glosario.pdf. (Consulta: 21 de agosto de 2004).

Páez, I. (1992) Gestión de la inteligencia, aprendizaje tecnológico y modernización del trabajo informacional. Retos y oportunidades. Caracas: Instituto de Estudios del Conocimiento.

Peña, T y Pirela, J. (2002) "Incorporación de las tendencias en educación superior del siglo XXI en el currículo de la Escuela de Bibliotecología y Archivología de la Universidad del Zulia”, en Revista Interamericana de Bibliotecología. Vol. 25, No. 2. julio-diciembre de 2002. Medellín-Colombia.

Pineda, M. et al (2003) "La sociedad de la información como una sociedad en transición: Caracterización, tendencias y paradojas", en Revista de Ciencias Sociales. Vol IX, No. 2. Mayo-Agosto de 2003. Universidad del Zulia. Facultad de Ciencias Económicas y Sociales.

Sánchez-Vegas, S. (2003) La Gerencia de Tecnologías de Información y Comunicación en Contextos Organizacionales: de la Arquitectura de la Información a la Arquitectura del Conocimiento. Conferencia dictada en la Facultad de Humanidades y Educación de la Universidad del Zulia, el 10 de junio de 2003.

Silvio, J. (1993) "La comunicación del conocimiento en un nuevo contexto tecnológico", en Silvio, J (Comp) Una nueva manera de comunicar el conocimiento. Ob. Cit. p.p. 13-28.

Sodré, M. (1998) Reinventando la cultura. La comunicación y sus productos. Barcelona-España: Gedisa.

_- (2000). "Entrevista”, en Revista Fronteiras. Vol. 2 - No. 1. Revista del Programa de Postgrado em Ciências da Comunicação da Unisinos. 
Tapscott, D. (1999) “Promesas y peligros de la tecnología digital”, en Revista de Tecnología de la Información. Maracaibo: Biblioservice.

Torrado, M. (2000). "Educar para el desarrollo de las competencias: una propuesta para Reflexionar", en Universidad Nacional de Colombia (comp.). Competencias y proyecto pedagógico. Bogotá: Unibiblos: 31-54.

Vásquez, J., Aznarán, R., Pérez, A. y Alvarado, E. (1999). Las ciencias básicas de la competencia clínica. Trujillo: UNI.

Vinent, M. (2000). “¿Qué significa aprender? Un punto de vista sobre el concepto de competencia”, en Universidad Nacional de Colombia (comp.). Competencias y proyecto pedagógico. Bogotá: Unibiblos: 55-76.

Wolton, D. (2000) Sobrevivir a Internet. Barcelona-España: Gedisa. 